\title{
Performance Evaluation of RDT, Light Microscopy, and PET-PCR for Detecting P. Falciparum Malaria Infections in the 2018 Zambia National Malaria Indicator Survey
}

Mulenga C. Mwenda

PATH

Abebe A. Fola

Purdue University

llinca I. Ciubotariu

Purdue University

Conceptor Mulube

PATH

Brenda Mambwe

PATH

Rachael Kasaro

PATH

Moonga B. Hawela

Zambia National Malaria Elimination Centre

Busiku Hamainza

Zambia National Malaria Elimination Centre

John M. Miller

PATH

Giovanna Carpi

Purdue University

Daniel James Bridges ( $\nabla$ dbridges@path.org )

PATH https://orcid.org/0000-0002-9424-713X

\section{Research}

Keywords: Diagnostics, P. falciparum, malaria, survey, prevalence, light microscopy, PCR, RDT

Posted Date: April 27th, 2021

DOI: https://doi.org/10.21203/rs.3.rs-449689/v1 
License: (c) (i) This work is licensed under a Creative Commons Attribution 4.0 International License. Read Full License 


\section{Abstract}

\section{Background}

Zambia continues to advance on the path to elimination with significant reductions in malaria morbidity and mortality. Crucial components that have contributed to progress thus far and are necessary for achieving the national malaria elimination goals include properly identifying and treating all malaria cases through accurate diagnosis. This study sought to compare and assess the diagnostic performance of RDTs and LM with PET-PCR as the gold standard using 2018 MIS data across Zambia to better understand diagnostic accuracy metrics and how these vary across a transmission gradient.

Methods

Cross-sectional samples collected in a nationally representative survey from 7 provinces in Zambia were tested for the presence of malaria parasites by light microscopy (LM), rapid diagnostic test (RDT) and the gold standard PET-PCR. Diagnostic performance was assessed including sensitivity, specificity, negativeand positive-predictive values across a wide malaria transmission spectrum. Diagnostic accuracy metrics were measured, and statistically significant differences were calculated between test methods for different outcome variables.

Results

From the individuals included in the MIS, the overall prevalence of $P$. falciparum malaria was $32.9 \%$ by RDT, $19.4 \%$ by LM, and $23.2 \%$ by PET-PCR. Herein, we compared RDT and LM diagnostic performance against gold standard PET-PCR and found that LM had higher diagnostic accuracy than RDTs (91.3\% vs $84.6 \%$ respectively) across the transmission spectrum in Zambia. However, we found that the performance of both diagnostics was significantly reduced in low parasitemia samples. Consistent with previous studies, RDT diagnostic accuracy was predominantly affected by a high rate of false positives.

\section{Conclusion}

While LM performance is overall superior to RDTs, the additional requirements for its implementation such as high-quality staining, microscopes, and additional manpower, means that its application will remain at the health facility level. In contrast, while RDTs overestimate prevalence as they have a higher false positive rate, their ability to be rapidly deployed makes them an excellent community level tool. Overall, we found that the performance of both diagnostics was acceptable, and their use should be continued.

\section{Background}

Although significant progress has been made globally to reduce malaria burden through a coordinated international effort focused on vector control, accurate diagnosis and appropriate treatment, malaria continues to be a major global public health problem with devastating impacts on human health and 
livelihood, especially in sub-Saharan Africa [1]. In 2019, there were an estimated 229 million cases and 409,000 deaths recorded from malaria worldwide, with the World Health Organization (WHO) African Region accounting for approximately $94 \%$ of cases and deaths [2]. In the past few years, progress has plateaued with respect to reductions in malaria mortality rates and case incidences as put forth by the WHO Global Technical Strategy for Malaria 2016-2030 (GTS). This has raised concerns for continued success in countries on the path to malaria elimination [1,3]. Moreover, the current COVID-19 pandemic across the globe will likely affect the availability and distribution of key malaria control interventions and thus will impact malaria transmission dynamics landscape $[4,5]$. Thus, monitoring malaria prevalence and understanding the changing malaria transmission landscape as interventions are intensified is key to achieving the planned malaria elimination.

To this end, many countries throughout Africa conduct Malaria Indicator Surveys (MIS), i.e. comprehensive and nationally representative household surveys to collect key malaria data [6]. Measured indicators include the use of interventions like long-lasting insecticide-treated nets (LLINs) or indoor residual spraying (IRS), as well as individual information about malaria diagnosis. These MIS aim to measure intervention implementation in a respective country, monitor malaria and anaemia prevalence, and evaluate the impact of interventions. One country in which the MIS is conducted is Zambia, a malaria endemic country in sub-Saharan Africa, which has made significant progress towards reducing malaria morbidity and mortality over the past decade through integrated malaria control interventions. For instance, national MIS malaria parasite prevalence by microscopy among children under 5 decreased to 9\% in 2018 from 17\% recorded in 2015 [7, 8]. The Zambia MIS 2018, aimed to assess progress towards achieving the targets of the National Malaria Elimination Strategic Plan 2017-2021, and was conducted by the Zambia Ministry of Health through the National Malaria Elimination Centre (NMEC) with support from partners e.g. PATH Malaria Control and Elimination Partnership in Africa (MACEPA) and the United States President's Malaria Initiative [7]. MIS in Zambia are performed every 2 to 3 years at the end of the malaria transmission season in Zambia during the months of April and May. The 2018 MIS is the sixth MIS carried out in this country since 2006 [7].

In Zambia, the main malaria control interventions include vector control and effective case management [9]. With consistent use of LLINs and IRS, transmission can decline but this also can lead to increases in spatial heterogeneity of transmission intensity. For example, in Zambia malaria prevalence by light microscopy (LM) ranged from $0.0 \%$ in Southern province to $30.4 \%$ in Luapula province in 2018 [7]. Therefore, it is of great interest to understand the impact of changes in malaria prevalence on diagnostic performance and consequently case detection, particularly in such regions with heterogeneous transmission.

Currently, Zambia uses two main diagnostic tools for malaria parasite detection: the Plasmodium falciparum specific Histidine-rich protein 2 (HRP2)-based rapid diagnostic tests (RDTs) and light microscopy (LM) [10]. RDTs detect $P$. falciparum trophozoite-derived HRP2, which accounts for $98 \%$ of malaria cases and morbidity in Zambia and the region at large $[7,9,11]$. RDTs are easy to use, do not require specialized training, can be used by any caregiver e.g., community health workers, and are the 
main diagnostic tool used in community management of malaria by both passive and active case detection [12-14]. In contrast, LM can identify and quantify the presence of multiple malaria species, but requires trained microscopists and additional infrastructure e.g., staining facilities, microscopes and electricity $[15,16]$. As a result, $L M$ is difficult to implement in a rural setting. Finally, it is worth noting that WHO guidelines suggest at least 100 fields of view need to be examined before declaring a slide negative. In low prevalence and pre-elimination settings where there are very few infections to identify this will translate to very low throughput.

Molecular methods e.g., photo-induced electron transfer polymerase chain, can detect parasitaemia below LM and RDTs limit of detection (LOD), making them excellent gold standards to assess diagnostic performance [17-19]. Like LM, multiple Plasmodium species can be simultaneously identified with PETPCR, and in areas of low transmission that are approaching elimination, these other malaria species may become more relevant $[17,20,21]$.

As Zambia continues to progress toward malaria elimination, it is essential to diagnose all malaria cases accurately to enable appropriate treatment. Understanding how RDTs, LM, and PET-PCR diagnostics compare across the Zambian transmission gradient will be crucial to achieving this goal. This study therefore aimed to assess the diagnostic performance of RDTs and LM for diagnosis of $P$. falciparum malaria using PET-PCR as the gold standard. Understanding how these three different diagnostics perform and quantifying diagnostic accuracy will provide insights on prevalence of malaria infection and how these metrics vary according to transmission intensity.

\section{Methods}

\section{Study Design}

In 2018, a MIS was performed that randomly surveyed a total of 4,177 households across 179 standard enumeration areas from ten provinces in Zambia [7], testing all children under the age of 5 (except Western province where children up to 9 years old were included) with an RDT (SD Bioline malaria Ag pf, Standard Diagnostics Inc., Republic of Korea) and collecting a thick smear microscopy slide and dried blood spot (DBS). The survey used a nationally representative two-stage stratified clustering sampling strategy to get country representative samples for the accurate estimation of malaria prevalence and other indicators across Zambia. High transmission provinces such as Luapula and Western were oversampled to better capture the wide range of prevalence. The MIS was conducted from mid-April to late May 2018 which was expected to be the time of peak prevalence of parasitaemia and severe anaemia nationwide as well as the timing for all previous MIS in Zambia. Three provinces with less than 20 positive RDTs (Central, Lusaka and Southern), and samples missing PET-PCR, RDT and LM results were excluded from the final data analysis. A total of 3153 DBS samples fulfilled the inclusion criteria from seven provinces across Zambia (Fig. 1). 


\section{Laboratory Methods}

\section{Sample and Data Collection}

Before performing the MIS, training was provided for health workers on how to perform an RDT, prepare a DBS and thick smear slide as well as how to interview community members and collect survey responses [7]. RDT testing was performed according to manufacturer recommendations. Slides, DBS, used RDTs and data were then transferred to the NMEC in Lusaka.

\section{Microscopy}

Thick smear microscopy slides were stained in 10\% Giemsa at the NMEC laboratory and read independently by two malaria microscopy-competent technicians. Arbitration of discordant results was performed by a third reader. A blood smear was considered negative when no parasites were detectable after examining at 100 high power fields. For positive smears, parasites were counted in reference to 200 white blood cells (WBC) when Plasmodium parasites were more than 10, or 500 WBC when Plasmodium parasites were less than 10, and then converted into a parasite concentration [22].

\section{DNA extraction}

DNA was extracted from a single 6-mm punch (equivalent to roughly $13 \mu \mathrm{L}$ of blood) from each DBS using the QIAamp DNA mini kit (QIAGEN, Hilden, Germany) and eluted in $100 \mu \mathrm{L}$ of Elution buffer. Punchers were cleaned by making 5 blank punches on clean filter paper between samples. A subset of blank punches was extracted for testing to ensure that there was no cross-over between samples. Extracted DNA was stored at $4^{\circ} \mathrm{C}$ for immediate analysis or at $-20^{\circ} \mathrm{C}$ for longer term storage.

\section{PCR Detection}

Extracted parasite DNA was detected by real-time PET-PCR, on a LightCycler 480 (Roche, Basel, Switzerland). In brief, all samples were tested in duplicate $(5 \mu \mathrm{L}$ of template, equivalent to $0.7 \mu \mathrm{L}$ of whole blood) in a duplex reaction with Plasmodium spp. and Plasmodium falciparum primers labelled with FAM and HEX fluorophores, respectively, as previously described [20, 23, 24]. Samples were scored positive when both replicates had cycle threshold values of $<40$ and standard deviation of $<2$. A limiting dilution series of 3D7 P. falciparum genomic DNA (MRA-151G, ATCC, Manassas, VA), obtained through BEI Resources, National Institute of Allergy and Infectious Diseases, National Institutes of Health, contributed by David Walliker, of a known parasitemia was assayed three times in duplicate by PET-PCR. The standard curves generated from this series established a comparable LOD, as previously published [9], and were used to determine parasitaemia.

\section{Statistical analysis}

Number of DBS samples per province were counted and compiled using dplyr and tidyverse function $\mathrm{R}$ package $[25,26]$. Using PET-PCR as the 'gold standard', diagnostic metrics (Sensitivity, Specificity and Predictive values) were calculated using the epi.test function ('epiR' version 1.0-15 R package) [27]. 
Figure visualization and statistical analyses (Mann-Whitney $U$ test was used to measure differences among two groups) were performed using GraphPad Prism (version 7.0.1 for Mac, GraphPad Software, San Diego, California, USA, www.graphpad.com) and a $P$ value of $\leq 0.05$ was considered statistically significant.

\section{Results}

\section{Descriptive statistics}

Overall, 32.9\% (1038 /3152), 19.4\% (535/2758), and 23.2\% (733/3153) of study participants were positive for $P$. falciparum by RDT, LM, and PET-PCR respectively across seven provinces. $17.0 \%$ (537/3152) of RDT results and $9.9 \%$ (274/2758) of LM results were discordant (reported as either falsely positive or falsely negative) when compared to PET-PCR (Fig. 2). RDTs identified more false positives, while LM identified more false negatives (FN), although the overall number of $F N$ samples was relatively low. Note that the variation RDT vs. LM total results was due to the large number of samples that were not tested by LM and thus excluded from the final data analysis.

Diagnostic prevalence per province was highly heterogenous as expected given the transmission gradient across Zambia. Specifically, RDT prevalence ranged from $25.4 \%-44.7 \%$, LM from $11.3 \%-32.6 \%$, and PET-PCR from 15.4\% - 38.0\% (Fig. 3A). Overall, however, there was a positive correlation between provincial prevalence (Fig. $3 \mathrm{~B}$ ) across the three methods indicating agreement between these three diagnostic methods to estimate $P$. falciparum malaria prevalence and burden across the full transmission gradient in Zambia.

\section{Diagnostic performance}

Diagnostic sensitivity, (the probability that a test result will be positive when the disease is present) and specificity (the probability that a test result will be negative when the disease is not present) for RDTs showed higher sensitivity $84 \%(95 \% \mathrm{Cl}=81-87)$, but lower specificity $83 \%(95 \% \mathrm{Cl}=81-84)$ when compared to $\mathrm{LM}$ at $71 \%(95 \% \mathrm{Cl}=67-74)$, and $96 \%(95 \% \mathrm{Cl}=95-97)$ respectively. We also measured Positive Predictive Value (PPV) (the probability that the disease is present when the test is positive) and Negative Predictive Value (NPV) (the probability that the disease is not present when the test is negative) and found that RDTs had very low PPV 59\% (95\% Cl=56-62) compared to LM at 84\% (95\% $\mathrm{Cl}=81-87)$ (Table 1$)$.

Table 1. Comparison of RDT and LM diagnostic metrics across Zambia. PPV: positive predictive value; NPV: negative predictive value. 


\begin{tabular}{|llllllll|}
\hline & Positive & Negative & Total & $\begin{array}{l}\text { Sensitivity } \\
(95 \% \mathrm{Cl})\end{array}$ & $\begin{array}{l}\text { Specificity } \\
(95 \% \mathrm{Cl})\end{array}$ & $\begin{array}{l}\text { PPV } \\
(95 \% \mathrm{Cl})\end{array}$ & $\begin{array}{l}\text { NPV } \\
(95 \% \mathrm{Cl})\end{array}$ \\
\hline Positive & 617 & 421 & 1038 & $84 \%$ & $83 \%$ & $59 \%$ & $95 \%$ \\
\hline Negative & 116 & 1998 & 2114 & & & & \\
\cline { 1 - 3 } & 733 & 2419 & 3152 & $(81-87)$ & $(81-84)$ & $(56-62)$ & $(93-95)$ \\
\hline Positive & 449 & 86 & 535 & 70 & 96 & 84 & 92 \\
Negative & 188 & 2035 & 2223 & & & & \\
\hline Total & 637 & 2121 & 2758 & $(67-74)$ & $(95-97)$ & $(81-87)$ & $(91-93)$ \\
\hline
\end{tabular}

Diagnostic accuracy, calculated as the proportion of true positive and true negatives among all individuals tested, was consistently higher for LM (91.3\%) vs RDT (84.6\%) across all seven provinces. Furthermore, there was no obvious variation in diagnostic accuracy with prevalence.

Other metrics found that LM had a statistically significantly higher specificity (range $=94-98 \%$ ) compared to that of RDT (range $=68-85 \%$ ). In contrast, RDT showed high sensitivity (range $=76-96 \%$ ) but very low PPV (range $=36-75 \%$ ) compared to the sensitivity of LM (range = 54-78\%), PPV (range $=71-90 \%$ ) (Fig. 5). For both diagnostics, while specificity and NPV remained similar across the prevalence range, both PPV and sensitivity appeared to decline as prevalence reduced (Fig 5).

\section{Effect of parasite density}

A total of 116 false negatives i.e., PET-PCR positive / RDT negative were identified. The parasitaemia in these samples $($ median $=7.31$ parasites $/ \mu \mathrm{L})$ was significantly lower $(P<0.001)$ than the parasitaemia (median $=106.1$ parasites $/ \mu \mathrm{L}$ ) in true positive $(\mathrm{TP})$ samples, i.e., PET-PCR positive / RDT positive (Fig. 6A). A similar difference was observed with the $188 \mathrm{LM}$ false negatives, whose parasitaemia (median = 5.65 parasites $/ \mu \mathrm{L})$ was significantly lower $(\mathrm{P}<0.001)$ than the parasitemia in LM true positives (median $=197$ parasites $/ \mu \mathrm{L}$ ) (Fig. 6B). Correlation analysis between diagnostic metrics and parasite prevalence revealed a positive statistically significant correlation for both RDT PPV $(r=0.85$, $P$. value $=0.009)$ and LM PPV $(r=0.75$, $P$. value $=0.021)$ respectively. No other statistically significant correlations between other diagnostic metrics (Sensitivity, Specificity and NPV) and parasite prevalence were identified.

\section{Discussion}

Malaria was placed at the forefront of the global public health agenda in 2000 when the Roll Back Malaria Partnership announced a commitment to halving malaria cases by 2015 [28]. A decade later, a $21 \%$ reduction in the malaria burden (clinical cases) had been achieved, with elimination [1] and even eradication now being pursued $[28,29]$. Underpinning these aims is the need for early, accurate diagnosis and effective treatment of infections [30], to reduce onward transmission and prevent the development of 
severe malaria [1]. Ensuring that diagnostics continue to perform well, through regular assessments in all transmission settings, is critical for successful disease management, surveillance, and reaching targets such as elimination [31]. The detection of Plasmodium antigens using RDTs are widely used for malaria diagnosis. They are cheap, do not require advanced sample preparation or specialized training, and rapidly generate results [13]. This combination has transformed malaria case management from an emphasis on symptomatic presumptive diagnosis to one focused on diagnostic confirmation [3]. RDTs are now also used in active case detection (ACD) to identify infections in the community both as an intervention and as a means to assess changes in prevalence [32]. However, the sensitivity and specificity of RDTs varies significantly between commercial providers [33-35]. We therefore compared RDT and LM diagnostic performance against PET-PCR as a gold standard across a transmission gradient in Zambia with samples collected in a nationally representative MIS in 2018.

While both diagnostics performed well, overall LM had a higher diagnostic accuracy (91.3\%) than RDTs (84.6\%), supporting the historical use of LM as the gold standard diagnostic [1]. In general, this discrepancy was due to RDTs recording a high number of false positives. This tendency to over-estimate malaria prevalence can be seen in Fig. 3b where RDT prevalence is consistently higher ( $10 \%)$ than PETPCR prevalence, as well as in the low RDT PPV (range $=36-75 \%$ ) compared to LM PPV (range $=71-$ $90 \%$ ). It is unclear from this study whether this relationship would hold below $15 \%$ true prevalence, but it seems unlikely that in near elimination settings there would be an $\sim 10 \%$ overestimate. This overestimation has been documented in other studies [36], and is most likely due to persistent antigenaemia post-infection, or to a lesser extent cross reactivity with other infections or autoantibodies [37, 38]. To investigate this observation further, we looked at the proportion of false positives and true negatives selfreporting having had a fever or taking drugs for a fever in the past two weeks. In these two groups, 33.3\% $(n=421)$ of false positives vs $17.8 \%(n=356)$ of true negatives reported fever / drugs taken in past two weeks. The elevated reporting in the false positive group potentially suggests that this group had a higher prevalence of malaria infection that either resolved by itself or was cleared with an antimalarial drug, either of which would lead to a false positive outcome. While not desirable, at the population level unnecessarily treating an additional $10 \%$ of the population is arguably better than missing $10 \%$ of the infected population. While this would translate to the consumption of additional treatments, current antimalarials have excellent safety profiles so is unlikely to unduly affect the cost-benefit analysis. Furthermore, if treatments are long-lived they could act as a prophylaxis. More worryingly, is the chance of false negative diagnostic results. In this case, an infection, and therefore the chance to break the chain of transmission, is missed. Both diagnostics perform well in this regard (Fig. 2), but RDTs have a noticeably lower FN rate (3.68\%) compared to LM (6.81\%). Similarly, LM prevalence was consistently below the PCR prevalence suggesting that this diagnostic is more likely to miss infections (Fig. 3b).

A key question this study set out to address was how diagnostic performance varies across a wide transmission / prevalence range, and in the main, the performance was consistent (Fig. 4). However, it does appear that sensitivity and PPV reduces as prevalence decreases, and more markedly with RDTs vs LM. This may be due to the diagnostics inherent LOD, i.e., the concentration of the target analyte that must be present for a diagnostic to be able to detect it. For symptomatic infections, that are generally 
characterized by high parasitaemias, a high LOD is unlikely to affect diagnostic performance. However, in ACD where asymptomatics with very low-density infections are likely to be encountered, the LOD could be critical in determining the success or failure of ACD as an intervention. As expected, the sensitivity of both LM and RDTs reduced as parasitaemia decreased (Fig. 6) suggesting that in settings where there is a high proportion of infections below the diagnostics LOD, e.g. pre-elimination, more sensitive diagnostic tools will be required to find the last asymptomatic infections $[39,40]$.

Overall, our findings from this study suggest that, to more accurately monitor malaria transmission dynamics, countries in pre-elimination settings require more sensitive diagnostic tools to detect asymptomatic and low parasitaemia cases. Molecular techniques such as nested polymerase chain reaction, quantitative real-time PCR, and ligase detection reaction fluorescent microsphere assay have been developed and have shown greater sensitivity for a broad range of parasitaemias [41, 42]. However, few molecular methods are commonly used in malaria endemic countries as they require a healthy capital budget, advanced laboratories, and skilled workforce. There have been many attempts to develop simplified molecular tools for malaria diagnosis appropriate for low-resource countries [43], e.g. loopmediated isothermal amplification (LAMP) is a molecular point-of-care test with high specificity and sensitivity (5 parasites/ $\mu$ l of blood) well below the LOD for LM/RDT $[44,45]$. Serological diagnostic assays (serosurveillance tools) that detect active or latent infection as well as past exposure may help to assess the malaria burden at a community level more accurately especially in low transmission settings where infections are rare, and surveys therefore require large sample sizes to confidently calculate prevalence [46-48].

This study had a number of limitations. Firstly, the MIS sampling design limited enrollment to children and we therefore could not evaluate diagnostic performance outside this age group. Moreover, there was a marked variation in sample size per province with overestimation of samples from some high transmission areas. This study also was not able to address treatment status of study subjects and other non-febrile illnesses among children to assess longevity of Plasmodium antigens after treatment and contribution of non-malaria illness on RDT false positivity.

\section{Conclusions}

This $P$. falciparum malaria diagnostic performance evaluation within the nationwide 2018 Zambia Malaria Indicator Survey supports the utility of malaria RDTs for community screening based on their low rate of false negatives and their ease of implementation. In contrast, LM remains a superior diagnostic method for the confirmation of active infections, and accurately treating patients. Notably, the parasitaemia of an infection is one of the main determining factors impacting diagnostic performance and therefore accurate determination of malaria parasite prevalence. Our findings further suggest that to improve malaria prevalence estimates and case detection in low transmission settings, more sensitive molecular diagnostic tools to detect asymptomatic and low parasitaemia infections may be necessary to achieve and sustain malaria elimination. 


\section{Abbreviations}

ACD Active Case Detection

DBS Dried Blood Spot

FN False Negative

FP False Positive

HRP2 Histidine Rich Protein 2

IRS Indoor Residual Spraying

LM Light Microscopy

LLIN Long Lasting Insecticidal Nets

MIS Malaria Indicator Survey

NMEC National Malaria Elimination Centre

NPV Negative Predictive Value

PPV Positive Predictive Value

RDT Rapid Diagnostic Test

TN True Negative

TP True Positive

WBC White Blood Cell

WHO World Health Organisation

\section{Declarations}

\section{Ethics approval and consent to participate}

Written informed consent was obtained from all study participants or their parents or legal guardians.

The study was approved by the University of Zambia Biomedical Research Ethics Committee (Ref 011-0218) and by the Zambian National Health Research Authority.

\section{Consent for publication}




\section{Availability of data and materials}

Raw metadata sets used and/or analyzed during the current study are available from the corresponding author on reasonable request and with permission from the NMEC.

\section{Competing interests}

The authors declare that they have no competing interests.

\section{Funding}

This work is based on research funded by the Bill \& Melinda Gates Foundation through a grant to PATH.

The findings and conclusions contained within are those of the authors and do not necessarily reflect positions or policies of the Bill \& Melinda Gates Foundation.

\section{Author's Contributions}

$\mathrm{AF}, \mathrm{GC}$ and $\mathrm{DB}$ conceived the study. BM, RK, MM and $\mathrm{CM}$ were responsible for DNA extraction and molecular diagnosis of Plasmodium falciparum infections. RK read microscopy slides. AF, IC and MC drafted the manuscript and undertook statistical analyses. All authors contributed to the writing of the manuscript and approved the submitted version of the manuscript. All authors read and approved the final manuscript.

\section{Acknowledgements}

The authors are grateful to the Zambian communities, particularly the volunteers and their families for providing blood samples during the survey. We would also like to thank the staff of the Zambia National Malaria Elimination Centre for their generous ongoing support, especially the field researchers who conducted the nationwide survey and the microscopists Tracy Phiri, Namwezi Kapembwa, Dorcas Nanyangwe, Benjamin Kabamba and Mr Mandanda who processed the blood slides. We would like to thank the Southern and Central Africa International Centers of Excellence for Malaria Research.

\section{References}

1. WHO. Global technical strategy for malaria 2016-2030. 2015 [cited 2020 November 22]; Available from: https://www.who.int/malaria/areas/global_technical_strategy/en. 
2. WHO. World malaria report 2020. 2020 [cited 2020 December 1]; Available from: https://www.who.int/teams/global-malaria-programme/reports/world-malaria-report-2020.

3. WHO. World malaria report 2019. 2019 [cited 2020 November 23]; Available from: https://www.who.int/publications/i/item/9789241565721.

4. Weiss DJ, et al. Indirect effects of the COVID-19 pandemic on malaria intervention coverage, morbidity, and mortality in Africa: a geospatial modelling analysis. Lancet Infect Dis. 2021;21(1):5969.

5. Zawawi A, et al. The impact of COVID-19 pandemic on malaria elimination. Parasite Epidemiology Control. 2020;11:e00187.

6. Program D, Malaria Indicator Surveys. 20182015 [cited 2021 February 12]; Available from: https://www.malariasurveys.org/.

7. Ministry of Health Zambia, M. Zambia National Malaria Indicator Survey Report. 20192015 [cited 2020 November 25]; Available from: https://www.path.org/resources/zambia-natl-malaria-indicatorsurvey-mis-2018/.

8. PMI. Zambia Malaria Operational Plan FY 2020. 2020 [cited 2020 November 22]; Available from: https://www.pmi.gov/docs/default-source/default-document-library/malaria-operationalplans/fy20/fy-2020-zambia-malaria-operational-plan.pdf?sfvrsn=6.

9. Sitali $L$, et al. Distribution of Plasmodium species and assessment of performance of diagnostic tools used during a malaria survey in Southern and Western Provinces of Zambia. Malaria Journal. 2019;18(1):130.

10. Ministry of Health. Zambia M. Guidelines for the Diagnosis and Treatment of Malaria in Zambia. 2017 [cited 2021 January 2]; 5:[Available from:

https://www.nmec.org.zm/s/NationalMalariaTreatmentGuidelines2017_Final20170917-1.pdf.

11. Howard RJ, et al. Secretion of a malarial histidine-rich protein (Pf HRP II) from Plasmodium falciparum-infected erythrocytes. J Cell Biol. 1986;103(4):1269-77.

12. Shiff CJ, Premji Z, Minjas JN. The rapid manual ParaSight-F test. A new diagnostic tool for Plasmodium falciparum infection. Trans R Soc Trop Med Hyg. 1993;87(6):646-8.

13. Counihan H, et al. Community Health Workers Use Malaria Rapid Diagnostic Tests (RDTs) Safely and Accurately: Results of a Longitudinal Study in Zambia. The American Journal of Tropical Medicine Hygiene. 2012;87(1):57-63.

14. WHO. Towards quality testing of malaria rapid diagnostic tests: evidence and methods. 2006 [cited 2020 December 1]; Available from: https://www.who.int/malaria/publications/atoz/929061238X/en/.

15. Chiodini PL. Malaria diagnostics: now and the future. Parasitology. 2014;141(14):1873-9.

16. Mathison BA, Pritt BS. Update on Malaria Diagnostics and Test Utilization. J Clin Microbiol. 2017;55(7):2009-17. 
17. Chishimba S, et al. Prevalence of Plasmodium falciparum and Non-falciparum Infections by PhotoInduced Electron Transfer-PCR in a Longitudinal Cohort of Individuals Enrolled in a Mass Drug Administration Trial in Southern Province, Zambia. Am J Trop Med Hyg. 2020;103(2):82-9.

18. Erdman LK, Kain KC. Molecular diagnostic and surveillance tools for global malaria control. Travel Med Infect Dis. 2008;6(1-2):82-99.

19. Mens $\mathrm{P}$, et al. Is molecular biology the best alternative for diagnosis of malaria to microscopy? A comparison between microscopy, antigen detection and molecular tests in rural Kenya and urban Tanzania. Trop Med Int Health. 2007;12(2):238-44.

20. Lucchi NW, et al. Molecular Diagnosis of Malaria by Photo-Induced Electron Transfer Fluorogenic Primers: PET-PCR. PLOS ONE. 2013;8(2):e56677.

21. Laban NM, et al. Comparison of a PfHRP2-based rapid diagnostic test and PCR for malaria in a low prevalence setting in rural southern Zambia: implications for elimination. Malaria Journal. 2015;14(1):25.

22. WHO. Malaria Parasite Counting. Malaria Microscopy Standard Operating Procedure - MM-SOP-09 2016 [cited 2020 December 1]; Available from: https://apps.who.int/iris/bitstream/handle/10665/274382/MM-SOP-09-eng.pdf? sequence $=14$ \&isAllowed $=y$.

23. Akerele $D$, et al. Molecular diagnosis of Plasmodium ovale by photo-induced electron transfer fluorogenic primers: PET-PCR. PLOS ONE. 2017;12(6):e0179178.

24. Lucchi NW, et al. PET-PCR method for the molecular detection of malaria parasites in a national malaria surveillance study in Haiti, 2011. Malaria Journal. 2014;13(1):462.

25. Wickham H, François R, Müller K, dplyr: A Grammar of Data Manipulation. R package version 0.7.6. 2018.

26. Wickham H, et al. Welcome to the tidyverse. Journal of Open Source Software. 2019;4(43):1686.

27. Cartensen B, et al., Epi: A Package for Statistical Analysis in Epidemiology. R package version 2.42. 2020.

28. Alonso PL, et al. A Research Agenda to Underpin Malaria Eradication. PLOS Medicine. 2011;8(1):e1000406.

29. Rabinovich RN, et al. malERA: An updated research agenda for malaria elimination and eradication. PLOS Medicine. 2017;14(11):e1002456.

30. Atkinson J-A, et al. Operational research to inform a sub-national surveillance intervention for malaria elimination in Solomon Islands. Malaria Journal. 2012;11(1):101.

31. Tangpukdee N, et al. Malaria diagnosis: a brief review. Korean J Parasitol. 2009;47(2):93-102.

32. Larsen DA, et al. Population-wide malaria testing and treatment with rapid diagnostic tests and artemether-lumefantrine in southern Zambia: a community randomized step-wedge control trial design. Am J Trop Med Hyg. 2015;92(5):913-21.

33. Moody A. Rapid diagnostic tests for malaria parasites. Clin Microbiol Rev. 2002;15(1):66-78. 
34. Kilian AHD, et al. Comparison of two rapid, HRP2-based diagnostic tests for Plasmodium falciparum. Transactions of The Royal Society of Tropical Medicine Hygiene. 1997;91(6):666-7.

35. Pieroni $P$, et al. Comparison of the ParaSight ${ }^{T M}-F$ test and the ICT Malaria Pf $^{\text {TM }}$ test with the polymerase chain reaction for the diagnosis of Plasmodium falciparum malaria in travellers. Transactions of The Royal Society of Tropical Medicine Hygiene. 1998;92(2):166-9.

36. Ranadive N, et al. Limitations of Rapid Diagnostic Testing in Patients with Suspected Malaria: A Diagnostic Accuracy Evaluation from Swaziland, a Low-Endemicity Country Aiming for Malaria Elimination. Clin Infect Dis. 2017;64(9):1221-7.

37. Poti KE, et al. HRP2: Transforming Malaria Diagnosis, but with Caveats. Trends in Parasitology. 2020;36(2):112-26.

38. Dalrymple U, et al. How long do rapid diagnostic tests remain positive after anti-malarial treatment? Malar J. 2018;17(1):228-8.

39. McMorrow ML, Aidoo M, Kachur SP. Malaria rapid diagnostic tests in elimination settings-can they find the last parasite? Clin Microbiol Infect. 2011;17(11):1624-31.

40. Joanny F, et al. Limit of blank and limit of detection of Plasmodium falciparum thick blood smear microscopy in a routine setting in Central Africa. Malar J. 2014;13:234-4.

41. Mixson-Hayden T, Lucchi NW, Udhayakumar V. Evaluation of three PCR-based diagnostic assays for detecting mixed Plasmodium infection. BMC Res Notes. 2010;3:88-8.

42. Rosanas-Urgell A, et al. Comparison of diagnostic methods for the detection and quantification of the four sympatric Plasmodium species in field samples from Papua New Guinea. Malaria Journal. 2010;9(1):361.

43. Cordray MS, Richards-Kortum RR. Emerging nucleic acid-based tests for point-of-care detection of malaria. Am J Trop Med Hyg. 2012;87(2):223-30.

44. Notomi T, et al. Loop-mediated isothermal amplification of DNA. Nucleic acids research. 2000;28(12):E63-3.

45. Cook J, et al. Loop-mediated isothermal amplification (LAMP) for point-of-care detection of asymptomatic low-density malaria parasite carriers in Zanzibar. Malaria Journal. 2015;14:43-3.

46. Oviedo A, et al. Combination of Serological, Antigen Detection, and DNA Data for Plasmodium falciparum Provides Robust Geospatial Estimates for Malaria Transmission in Haiti. Sci Rep. 2020;10(1):8443.

47. Surendra $H$, et al. Using health facility-based serological surveillance to predict receptive areas at risk of malaria outbreaks in elimination areas. BMC Med. 2020;18(1):9.

48. Rogier $E$, et al. High-throughput malaria serosurveillance using a one-step multiplex bead assay. Malar J. 2019;18(1):402-2.

\section{Figures}




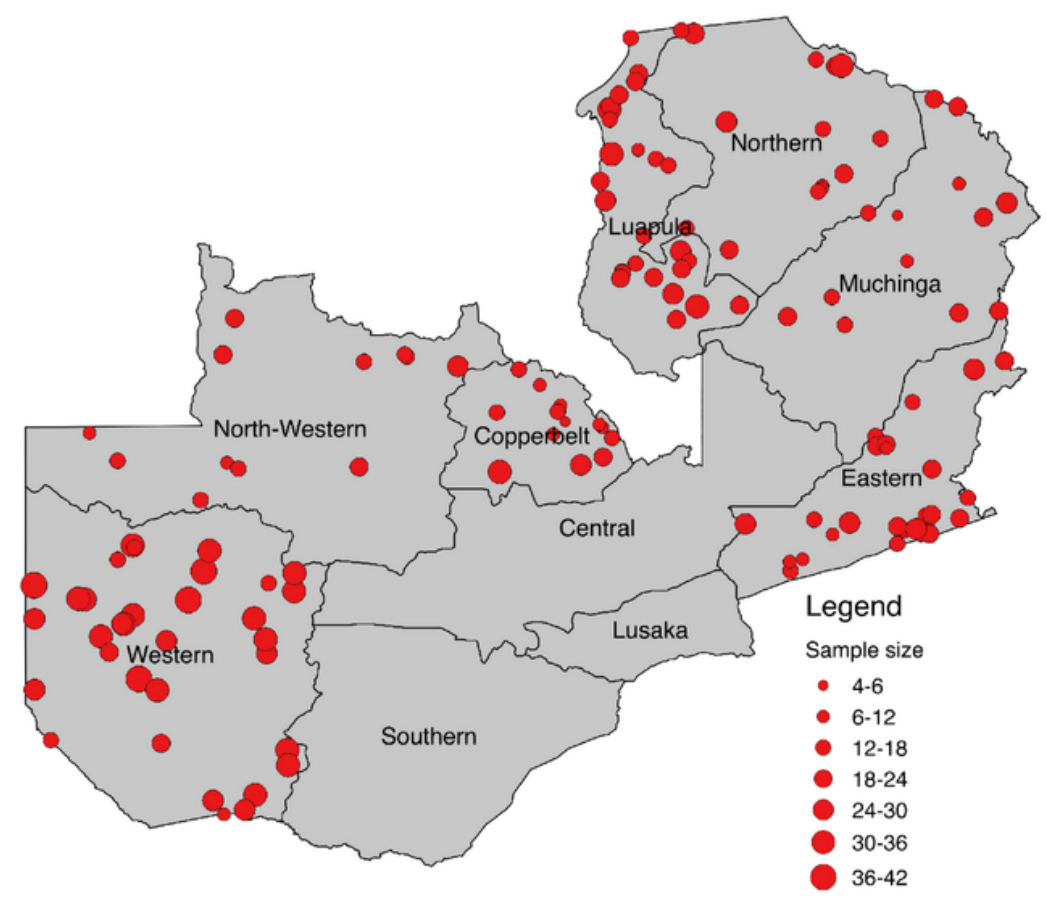

\section{Figure 1}

Map of Zambia showing 3153 DBS samples across seven provinces included in the diagnostic method comparison analysis. Sample collection locations or clusters (red circles) are shown proportional to sample size. Note: The designations employed and the presentation of the material on this map do not imply the expression of any opinion whatsoever on the part of Research Square concerning the legal status of any country, territory, city or area or of its authorities, or concerning the delimitation of its frontiers or boundaries. This map has been provided by the authors. 


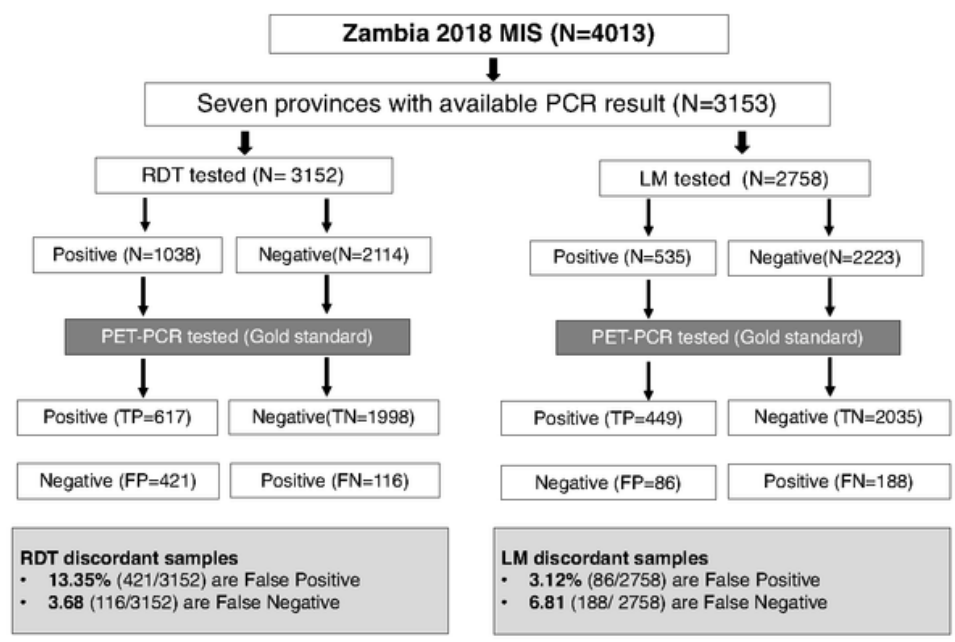

\section{Figure 2}

Schematic flow chart showing study participant and diagnostic tests discordance. PET-PCR $=$ PhotoInduced Electron Transfer Polymer Chain Reaction, RDT = Rapid Diagnostic Test, LM = Light Microscopy, $\mathrm{TP}=$ True Positive, $\mathrm{TN}=$ True Negative, FP = False positive, FN = False Negative 
A.

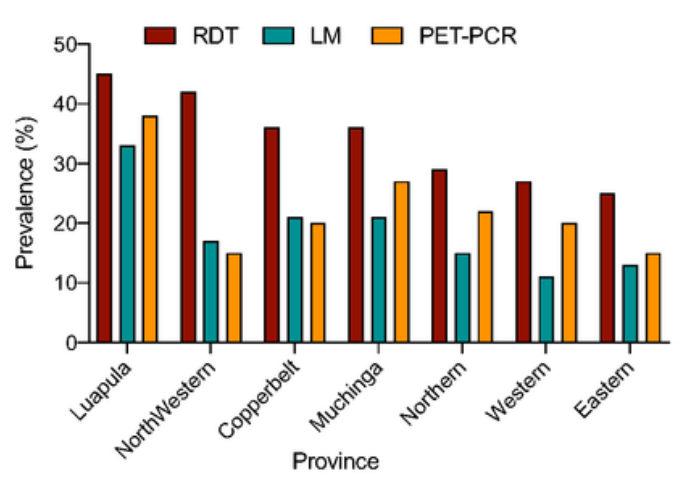

B.

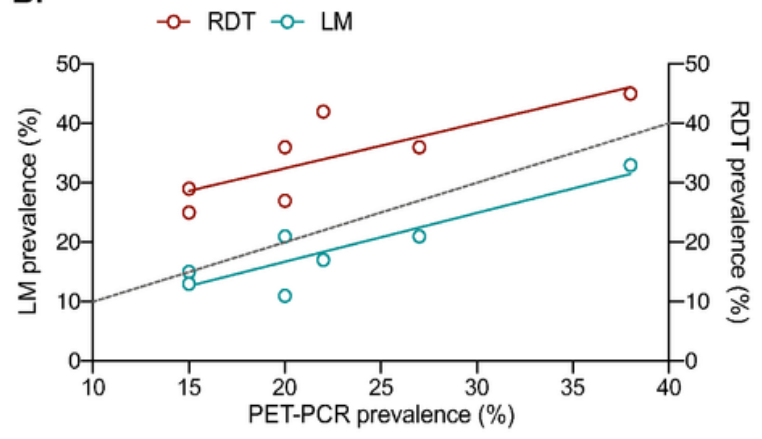

\section{Figure 3}

P. falciparum malaria prevalence and its comparison across provinces by the three diagnostic methods. A) Prevalence of P. falciparum malaria per province for each diagnostic assay, ranked in decreasing order of RDT prevalence. B) Correlation between RDT and LM prevalence against PET-PCR prevalence. Dashed line shows where $x=y$, i.e. perfect prevalence correlation between the PCR and LM or RDT diagnostics. 


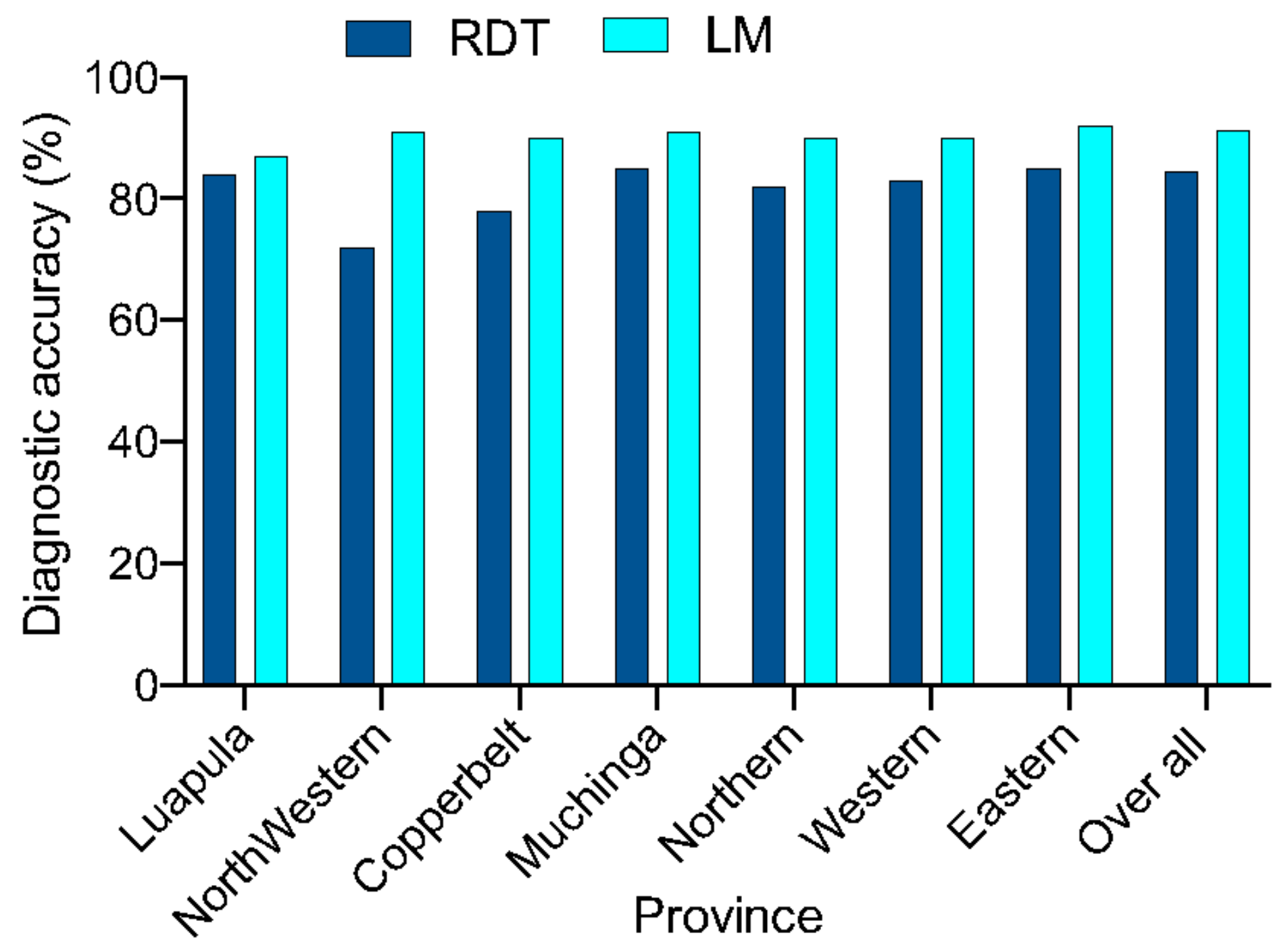

Figure 4

Comparison of diagnostic accuracy between RDT and LM for each province in Zambia. The diagnostic accuracy is the proportion of individuals who are true positive and true negative among all individuals tested. 

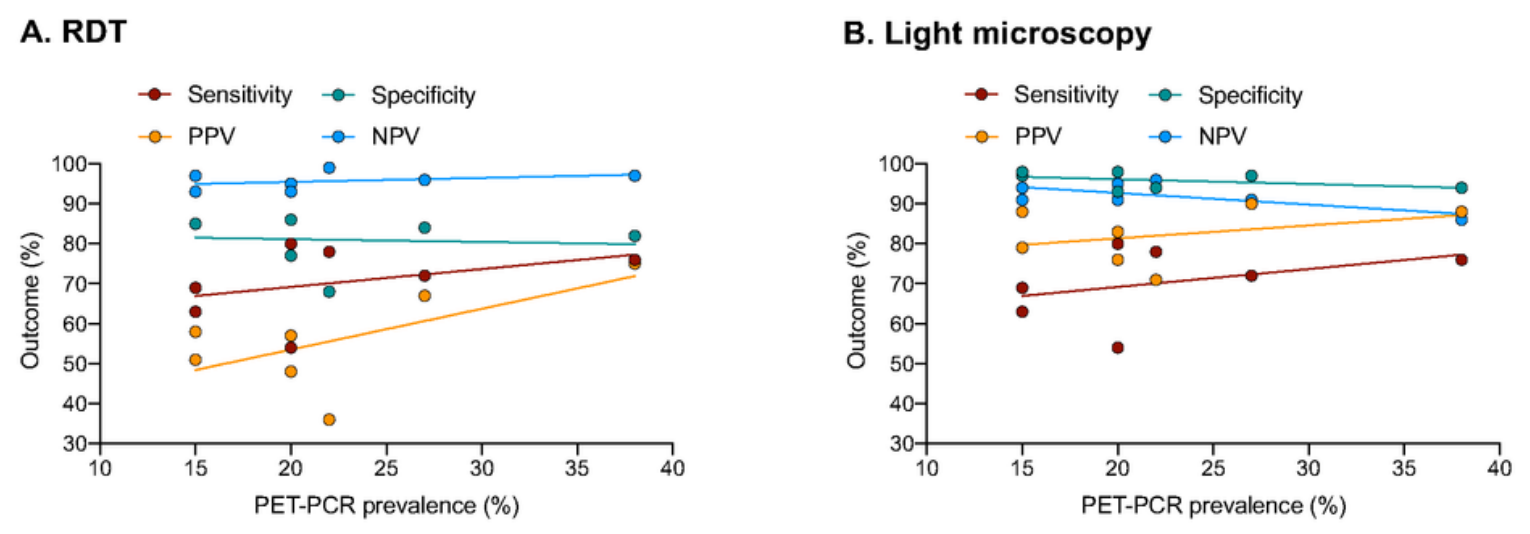

\section{Figure 5}

Diagnostic performance metrics of RDTs (A) and LM (B) across Zambia, using PET-PCR as the gold standard. PPV-positive predictive value, NPV - negative predictive value.

\section{A. RDT}

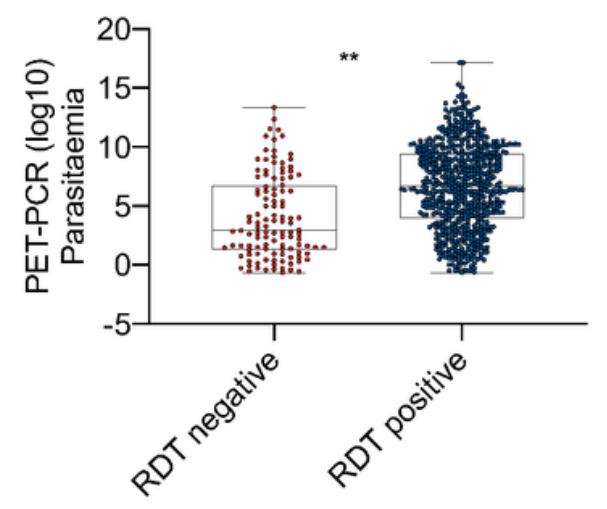

\section{B. Light microscopy}

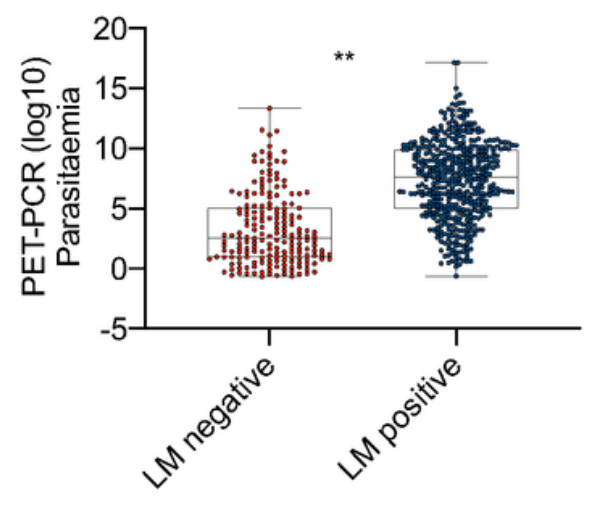


Figure 6

Comparison of parasitaemia for RDT (A) and LM (B) samples stratified by diagnostic result. A statistically significant difference in parasitaemia level between RDT/LM negative vs RDT/LM positive samples was identified. $* *=p<0.001$

\section{Supplementary Files}

This is a list of supplementary files associated with this preprint. Click to download.

- SupplementaryTable.docx 